\title{
Improved Joint Optimization Design for Wireless Sensor and Actuator Networks with Time Delay
}

\author{
Lihan Liu $\mathbb{D}^{1,2}$, Yuehui Guo $\mathbb{D}^{1},{ }^{1}$ Yang Sun $\mathbb{D}^{1},{ }^{1}$ Zhuwei Wang $\mathbb{D},{ }^{1}$ Enchang Sun $\mathbb{D}^{1},{ }^{1}$ \\ and Yanhua Sun ${ }^{1}$ \\ ${ }^{1}$ Faculty of Information Technology, Beijing University of Technology, Beijing 100124, China \\ ${ }^{2}$ School of Information, Beijing Wuzi University, Beijing, Beijing 101149, China \\ Correspondence should be addressed to Yang Sun; sunyang@bjut.edu.cn
}

Received 24 September 2021; Accepted 9 December 2021; Published 30 December 2021

Academic Editor: Xingsi Xue

Copyright (c) 2021 Lihan Liu et al. This is an open access article distributed under the Creative Commons Attribution License, which permits unrestricted use, distribution, and reproduction in any medium, provided the original work is properly cited.

\begin{abstract}
With the rapid development of wireless communication technology, the newest development of wireless sensor and actuator networks (WSANs) provides significant potential applications for various real-time scenarios. Currently, extensive research activities have been carried out in the field of efficient resource management and control design. However, the stability of the controlled plant and the efficiency of network resources are rarely considered collaboratively in existing works. In this paper, in order to enhance the control stability and improve the power consumption efficiency for the WSAN, a novel three-step optimization algorithm jointly designing the control strategy and transmission path routing is proposed when the time delay is considered. First, the minimum hop routing algorithm is used to obtain the set of candidate transmission paths. Then, the optimal control signals for each candidate transmission path can be iteratively derived with a backward recursion method. Finally, the best transmission path is determined under the optimal control strategy to achieve the joint optimization design. The effectiveness of the proposed joint optimization algorithm is verified by the simulations of the application in the power grid system.
\end{abstract}

\section{Introduction}

Wireless communication technology plays a vital role in various communication networks to promote the progress of modern science and technology [1-3]. It is believed that the emergence and development of the wireless technology are revolutionizing the traditional wired communication. Wireless sensor and actuator networks (WSANs), typically consisting of sensors, controllers, and actuators, is one of the most critical wireless communication applications [4]. With the characteristic of spatially distributed nodes, WSAN has the capability of information perception, transmission, analysis, and processing to meet the demands for both high reliability and low latency. Efficient information sharing and energy consumption management can be realized in the closed-loop feedback control network with proper resource allocation and transmission path routing. Currently, WSAN has already become an attractive research topic in many application areas, such as Internet of Thing (IoT), intelligent transportation, automotive industry, and smart healthcare [5-10].

WSAN takes advantage of the wireless network to provide information sharing, resource utilization, and plant control. However, there are still some challenges introduced especially with the increasing number of connected devices and sensor nodes [11-14]. One of the problems is the time delay caused by wireless communication which may significantly degrade the system performance and even cause instability [15]. Many works have been done to alleviate the influences of the delays. In [16], the network-induced short delay is analyzed for addressing the real-time system control problem. It uses the stochastic control theory to analyze the optimal state feedback for stabilization in discretetime domain. The authors in [17] study an optimal controller for network control systems to maintain stability under the long time delay caused by wireless communication. An 
overview makes a deep analysis of stability of linear systems with time-varying delays in [18]. Fog computing is introduced to minimize the delay for IoT applications in [19-20]. Moreover, in [21], a packet-based control law is proposed in networked control systems that explicitly compensates losses of the delay, data packet disorder, and data packet dropout based on Markov chain. Currently, the joint optimization design in wireless sensor networks has begun to attract more and more attention. A two-step algorithm is proposed in [22] when the real-time control and resource management are collaboratively considered.

In addition, the power consumption has gradually become another challenging problem [23-25]. In [26], two distributed local algorithms dynamically adjust the transmission power level per node and are proposed to take advantages in improving energy efficiency. The medium access control protocol in [27] is provided for efficiently reducing energy consumption with a two-radio architecture. In [28], an optimization method of controller and communication systems is proposed to minimize the power consumption for wireless networked control systems considering the imperfections of time delay and packet error. Joint resource allocation and power control are investigated to maximize the energy efficiency of device-to-device (D2D) communications in [29]. In [30], a power-based vehicle longitudinal control optimal algorithm is proposed to minimize energy consumption of the connected ecodriving system. In [31], the latency optimization for resource allocation is proposed in mobile edge computation offloading. Furthermore, the power consumption is considered a key indicator in many actual applications. Currently, in multihop wireless sensor network, a distributed power control and data scheduling algorithm based on a differential game framework is proposed in [32] to achieve effective use of the available harvested energy and balance the buffers of all sensor nodes.

Unfortunately, most of the existing works focus on either control strategy design or power consumption management in the WSAN. The overviews in [33-34] and our previous work [35] reveal potential benefits of jointly optimizing control strategy and power consumption. However, few studies have taken into account these two aspects simultaneously. In this paper, a novel joint optimization algorithm is proposed, which meets the requirements of the real-time control and power consumption reduction. The main contributions of this paper can be summarized as follows:

(i) In discrete-time domain, based on the control dynamics modeling and power consumption analysis, the optimization problem jointly considering the control stability and power consumption efficiency is formulated in the presence of the time delay

(ii) A novel three-step joint optimization algorithm is proposed. First, the set of candidate paths is obtained by using the minimum hop routing algorithm. Next, the optimal control strategy for each candidate path can be iteratively derived with a backward recursion method. Finally, the joint optimization design is real- ized through the best transmission path selection under the determined optimal control strategy

(iii) In particular, the minimum hop routing algorithm is obtained based on the strong correlation between the utility function of the WSAN and the networkinduced time delay, which is totally determined by the number of hops in the transmission path. In addition, the optimal control signal can be derived as a linear function of the current state information and previous control signals

The rest of this article is organized as follows. Section 2 gives the system model and problem description. Then, in Section 3, the joint optimal control design under the influence of time delay is derived. The simulation experiment and conclusion are presented in Section 4 and Section 5, respectively.

\section{System Model and Problem Formulation}

In this section, a typical WSAN model consists of the plant, sensor, actuator, controller, and spatially distributed network nodes as shown in Figure 1. The sensors adjacent to the controlled plant sample the state information at periodic intervals. The controller acts as a decision maker to arrange an optimal transmission path and generate control strategies to realize the closed-loop feedback control. Then, the control signals are sent along this transmission path to the actuators to ensure the desirable dynamic and steady-state response. However, the time delay induced by the shared wireless communication among the WSAN components has serious effects on the stability of real-time control application [35]. Considering time delay, the joint optimization design of WSAN is investigated in this paper to achieve both plant control stability and energy efficiency of the wireless sensor network.

2.1. Power Consumption Analysis. In general, there are several alternative transmission paths available in wireless sensor networks because of the inherent nature of distributed network structure. A simple dual-path case is shown in Figure 1 that the solid transmission path $(1 \longrightarrow 2 \longrightarrow$ controller $\longrightarrow 7 \longrightarrow 8$ ) and the dotted transmission path ( $1 \longrightarrow 3 \longrightarrow 4 \longrightarrow$ controller $\longrightarrow 5 \longrightarrow 6 \longrightarrow 8$ ) are two candidate paths. In particular, a given transmission path is depicted in Figure 2, where there are $m_{k}$ network nodes with the controller located at the $m_{k}^{c}$ th node.

Considering a given transmission path $k$, the transmission power consumption from the $j$ th network node to the $(j+1)$ th network node can be given by [36]

$$
P_{j, j+1}^{k}=\mu\|x\|^{2}+\kappa_{d}\|x\|^{2} d_{j, j+1}^{r}
$$

where $\|x\|$ denotes the amplitude of the signal $x, r$ is the signal attenuation factor, $d_{j, j+1}$ represents the transmission distance, and $\mu$ and $\kappa_{d}$ are system determined constants.

Then, in the $i$ th sampling interval $(i T,(i+1) T)$, the sensor-to-controller and the controller-to-actuator power 


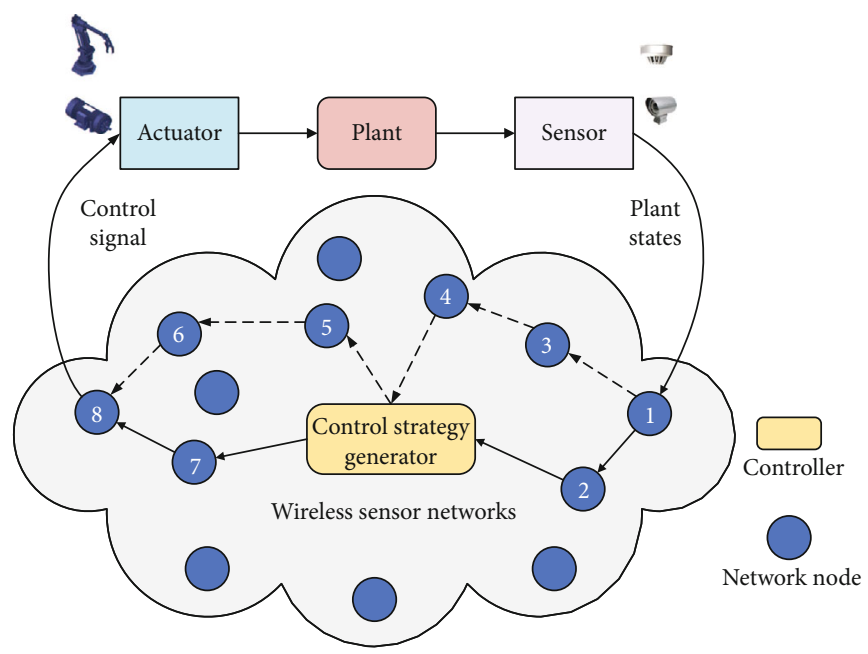

FIgURE 1: Structure of WSANs.

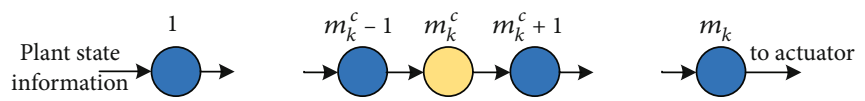

Figure 2: The $k$ th transmission path.

consumptions can be, respectively, expressed as [22].

$$
\begin{gathered}
P_{s c, i}^{k}=\sum_{j=1}^{m_{k}^{c}-1}\left(\mu\left\|s_{i}\right\|^{2}+\kappa_{d}\left\|s_{i}\right\|^{2} d_{j, j+1}^{r}\right), \\
P_{c a, i}^{k}=\sum_{j=m_{k}^{c}}^{m_{k}}\left(\mu\left\|u_{k, i}\right\|^{2}+\kappa_{d}\left\|u_{k, i}\right\|^{2} d_{j, j+1}^{r}\right),
\end{gathered}
$$

where $s_{i}$ is the sampled plant state and $u_{k, i}$ is the control signal generated based on the received $s_{i}$.

Thus, the total transmission power consumption of the entire control process is

$$
P_{\text {net }}^{k}=\sum_{i=0}^{J-1}\left(P_{s c, i}^{k}+P_{c a, i}^{k}\right)
$$

where $J$ is the number of sampling intervals in the control process.

2.2. Control System Modeling. In WSANs, the system dynamics in ith sampling interval can be formulated as [16]

$$
s_{i+1}=V_{i} s_{i}+W_{i 1} u_{k, i}+W_{i 2} u_{k, i-1} \text {, }
$$

where

$$
\begin{gathered}
V_{i}=e^{V T}, \\
W_{i 1}=\int_{0}^{T-\tau} e^{V t} d t W, \\
W_{i 2}=\int_{T-\tau}^{T} e^{V t} d t W,
\end{gathered}
$$

and here $V$ and $W$ are determined system parameters and $\tau$ is the network-induced time delay, which is influenced by many factors such as the sensor distribution, node size, network topology, and even signal transmission, processing, and reception.

In order to ensure the WSAN stability, the objective of the control strategy design is to minimize the system cost function as [37]

$$
P_{\text {cont }}^{k}=s_{J}^{T} B_{J} s_{J}+\sum_{i=0}^{J-1}\left(s_{i}^{T} B_{0} s_{i}+u_{k, i}^{T} C_{0} u_{k, i}\right),
$$

where $B_{J}, B_{0}$, and $C_{0}$ are determined system matrices.

2.3. Optimization Problem Formulation. Considering both power consumption efficiency and control stability of WSANs, the utility function of the joint optimization problem can be expressed as

$$
P_{J}^{k}=P_{\text {cont }}^{k}+\beta P_{\text {net }}^{k}
$$

where $\beta$ is a weight coefficient. 
Then, the utility function can be rewritten as

$$
P_{J}^{k}=s_{J}^{T} B_{J} s_{J}+\sum_{i=0}^{J-1}\left(s_{i}^{T} B s_{i}+u_{k, i}^{T} C u_{k, i}\right)
$$

where

$$
\begin{gathered}
B=B_{0}+\beta\left[\mu\left(m_{k}^{c}-1\right)+\kappa_{d} \sum_{j=1}^{m_{k}^{c}-1} d_{j, j+1}^{r}\right] I_{B}, \\
C=C_{0}+\beta\left[\mu\left(m_{k}-m_{k}^{c}+1\right)+\kappa_{d} \sum_{j=m_{k}^{c}}^{m_{k}} d_{j, j+1}^{r}\right] I_{C},
\end{gathered}
$$

and $I_{i}$ is an identity matrix with the same size as $i$.

Therefore, the objective of the joint optimization problem is to minimize the utility function through the transmission path routing and control strategy design, which is formulated as

$$
\begin{gathered}
\min _{\left\{u_{k, i}, k\right\}} P_{J}^{k}=s_{J}^{T} B_{J} s_{J}+\sum_{i=0}^{J-1}\left(s_{i}^{T} B s_{i}+u_{k, i}^{T} C u_{k, i}\right), \\
\text { s.t.s } s_{i+1}=V_{i} s_{i}+W_{i 1} u_{k, i}+W_{i 2} u_{k, i-1} .
\end{gathered}
$$

\section{Joint Optimization Algorithm Design}

In this section, a novel three-step algorithm is proposed to solve the joint optimization problem in (9). First, the candidate set of transmission paths is obtained by using the minimum hop routing algorithm. Then, for a given candidate transmission path, the control strategy can be derived in a backward recursion manner. Finally, the best transmission path selection under the optimal control strategy is determined.

In fact, it is difficult to directly solve the joint optimization problem (9). According to the principle of decoupling, the joint optimization problem can be decomposed into two subproblems: (S1) When the control strategy is designed, the joint optimization problem can be simplified to be a optimal path routing problem. (S2) When the transmission path is selected, the joint optimization problem can be converted to an individual control design problem. That is,

$$
\begin{gathered}
\mathrm{S} 1: \\
\min _{\left\{u_{k, i}^{*}, k\right\}} P_{J}^{k}=s_{J}^{T} B_{J} s_{J}+\sum_{i=0}^{J-1}\left(s_{i}^{T} B s_{i}+\left(u_{k, i}^{*}\right)^{T} C u_{k, i}^{*}\right), \\
\text { s.t. } s_{i+1}=V_{i} s_{i}+W_{i 1} u_{k, i}^{*}+W_{i 2} u_{k, i-1}^{*}, \\
\mathrm{~S} 2: \\
\min _{\left\{u_{k^{*}, i}, k^{*}\right\}} P_{J}^{k^{*}}=s_{J}^{T} B_{J} s_{J}+\sum_{i=0}^{J-1}\left(s_{i}^{T} B s_{i}+u_{k^{*}, i}^{T} C u_{k^{*}, i}\right), \\
\text { s.t.s } s_{i+1}=V_{i} s_{i}+W_{i 1} u_{k^{*}, i}+W_{i 2} u_{k^{*}, i-1},
\end{gathered}
$$

where $u_{k, i}^{*}$ and $k^{*}$ denote the optimal control strategy and the optimal transmission path routing, respectively.

In general, the transmission path routing and control strategy design can be addressed base on subproblems S1 and S2, respectively, and then iteratively converge to the joint optimal design. However, the iteration process usually has uncertain convergence and large computational complexity. Below, the further analysis is presented to simplify the iteration process.

3.1. Optimization Problem Transformation. In order to solve S1 in (11), a typical approach is the exhaustive search method to derive the best transmission path. However, it requires lots of computations, especially in a large-scale network. Therefore, a set of candidate paths needs to be determined first to reduce the computation burden.

Theorem 1. The subproblem S1 can be converted to be the transmission path selection problem with minimum hop count.

Proof. Based on the assumption in subproblem S1, the optimization problem can be simplified as the following optimal transmission path selection problem

$$
\min _{\left\{u_{k, i}^{*}, k\right\}} P_{J}^{k}\left(u_{k, i}^{*}\right)=s_{J}^{T} B_{J} s_{J}+\sum_{i=0}^{J-1}\left(s_{i}^{T} B s_{i}+\left(u_{k, i}^{*}\right)^{T} C u_{k, i}^{*}\right) .
$$

Then, the set of candidate transmission paths $\eta$ subject to the minimum utility function can be obtained as

$$
\eta=\arg \min _{\{k\}} P_{J}^{k}\left(u_{k, i}^{*}\right)
$$

In the WSAN, wireless communication may introduce time delays resulting in the system instability. Existing works [38-40] demonstrate the strong correlation between the delay and the utility function that a larger delay leads to an increase in the utility function, and vice versa. Thus, the minimum utility function can be transformed into the minimum delay problem as follows:

$$
\eta=\arg \min _{\{k\}}\left\{\tau_{k}\right\}
$$

where $\tau_{k}$ is the time delay of $k$ th transmission path.

Theoretically, the time delay mainly includes transmission and access delays, which is typically proportional to the hop count $[39,41]$. Therefore, the optimization problem in (15) can be equivalent to the minimum hop problem as

$$
\eta=\arg \min _{\{k\}} \mathrm{HC}_{k}
$$

where $\mathrm{HC}_{k}$ denotes the number of hops of a given transmission path. $\square$

3.2. Minimum Hop Routing Algorithm. In this subsection, the minimum hop routing algorithm is provided to efficiently reduce the computational complexity. First, the 
dynamic programming approach, as an optimization method of multistage decision-making, is used to transform the nonstandardized network into a the standardized one to provide a coherent and regular architecture. Then, the set of candidate transmission paths is derived based on the minimum hop routing algorithm.

In general, as shown in Figure 3, the sensor network without obvious decision-making stages is called the nonstandardized network. it is difficult to find the minimum hop routing directly with the increase in the number of sensor nodes. In order to search the path quickly, it is necessary to add virtual nodes to convert the network into a standardized sensor network. It can be seen from the Figure 3 that the number of hops from node $A$ to node $E$ can be 2 hops $(A \longrightarrow C \longrightarrow E)$ or 3 hops $(A \longrightarrow B \longrightarrow C \longrightarrow E)$, so a virtual child node $E 1$ equivalent to node $E$ can be added in the second decision-making stage as shown in Figure 4.

Based on the step above, the nonstandardized network problem can be successfully addressed. It is assumed that there are i sensor nodes during the $l$ th decision-making stage. Then, we can model the minimum hop count function in the $l$ th decision-making stage as follows:

$$
\operatorname{minHC}_{(l, i)}=\operatorname{minHC}_{(l-1, i)}+\operatorname{minHop}_{(l, i)} \text {, }
$$

where

$$
\operatorname{Hop}_{(l, i)}= \begin{cases}0, & \text { virtuallink } \\ 1, & \text { otherwise. }\end{cases}
$$

(1) $l=0$ : when $l=0$, the minimum hop count function in the source node is

$$
\operatorname{minHC}(0, i)=0
$$

(2) $l=1$ : identify and store the adjacent nodes with the smallest hop count in terms of (18), and the minimum hop count function when $l=1$ has

$$
\operatorname{minHC}(1, i)=\operatorname{minHop}(1, i)
$$

(3) $l=2, \cdots, n-1, n$ : here, $n$ is the total decision stages. We can obtain the set of candidate transmission paths with the minimum hop count as in (17). Finally, the set of candidate transmission paths can be derived as

$$
\eta=\arg \min _{\{k\}} H C_{(n, i)} \text {, }
$$

where $\{k\}$ is the set of available transmission paths in the standardized sensor network.

By using the proposed minimum hop routing algorithm summarized as in Algorithm 1, the set of candidate transmission paths to the optimization problem in (16) can be obtained. For example, as in Figure 4, there are three candidate transmission paths, namely, $A \longrightarrow C \longrightarrow D \longrightarrow F, A$ $\longrightarrow B \longrightarrow D \longrightarrow F$, and $A \longrightarrow C \longrightarrow E \longrightarrow F$.

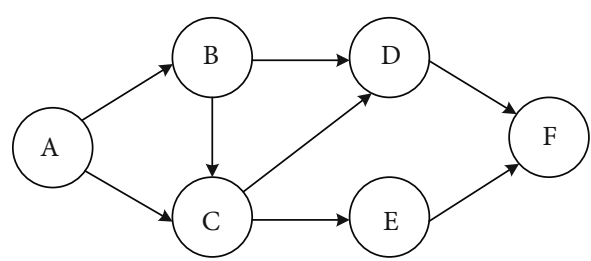

FiguRE 3: Nonstandardized sensor networks.

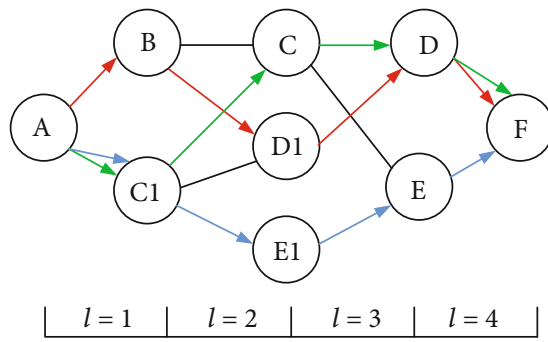

- Candidate path 1

— Candidate path 2

Candidate path 3

FIgURE 4: Standardized sensor networks.

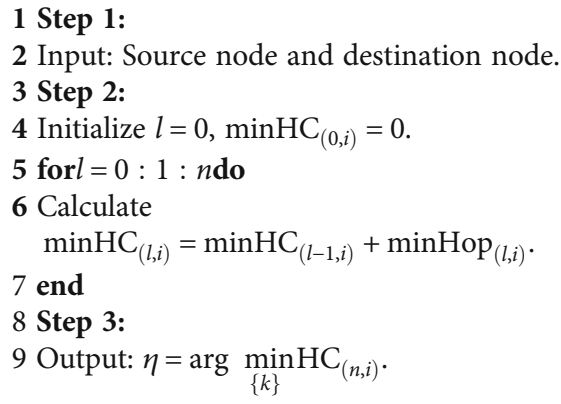

Algorithm 1: Minimum hop routing algorithm.

3.3. Optimal Control Design. For a given transmission path $\widehat{k} \in \eta$, the optimization problem in (13) can be equivalent to the following problem:

$$
\begin{gathered}
\min _{\left\{u_{\hat{k}, i}\right\}} P_{J}^{\widehat{k}}=s_{J}^{T} B_{J} s_{J}+\sum_{i=0}^{J-1}\left(s_{i}^{T} B s_{i}+u_{\widehat{k}, i}^{T} C u_{\widehat{k}, i}\right) \\
\text { s.t. } s_{i+1}=V_{i} s_{i}+W_{i 1} u_{\widehat{k}, i}+W_{i 2} u_{\widehat{k}, i-1} .
\end{gathered}
$$

Define a new state vector $f_{\widehat{k}, i}=\left[s_{i}^{T}, u_{k \wedge, i-1}\right]^{T}$, and then, the optimization problem in (22) can be rewritten as

$$
\begin{gathered}
\min _{\left\{u_{\widehat{k}, i}\right\}} P_{J}^{\widehat{k}}=f_{\widehat{k}, J}^{T} \bar{B}_{J} f_{\widehat{k}, J}+\sum_{i=0}^{J-1}\left[\begin{array}{l}
f_{k \wedge, i} \\
u_{k \wedge, i}
\end{array}\right]^{T}\left[\begin{array}{ll}
\bar{B} & 0 \\
0 & C
\end{array}\right]\left[\begin{array}{l}
f_{\widehat{k}, i} \\
u_{\widehat{k}, i}
\end{array}\right], \\
\text { s.t. } f_{\widehat{k}, i+1}=E_{i} f_{\widehat{k}, i}+F_{i} u_{\widehat{k}, i},
\end{gathered}
$$




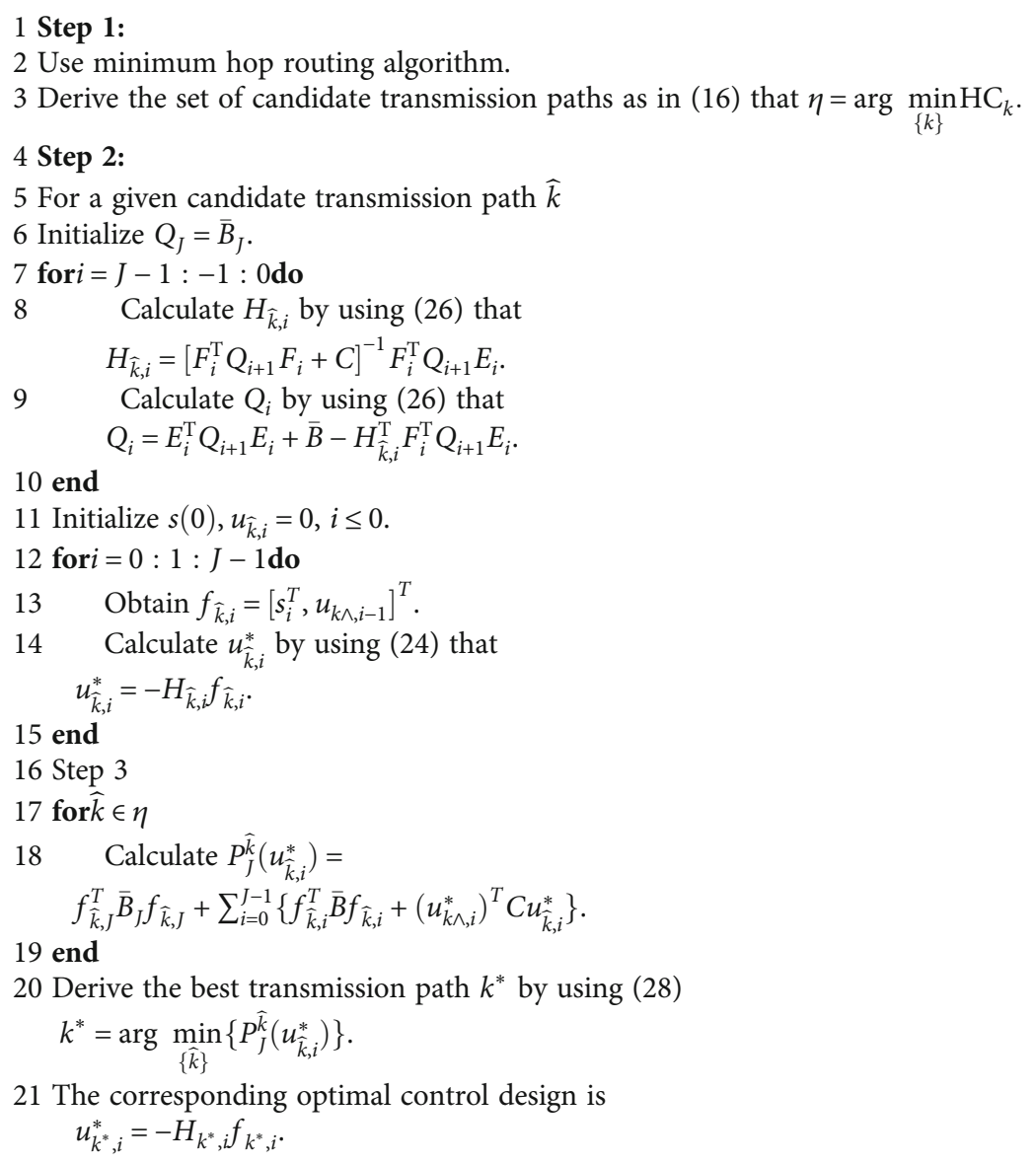

Algorithm 2: Three-step joint optimization algorithm.

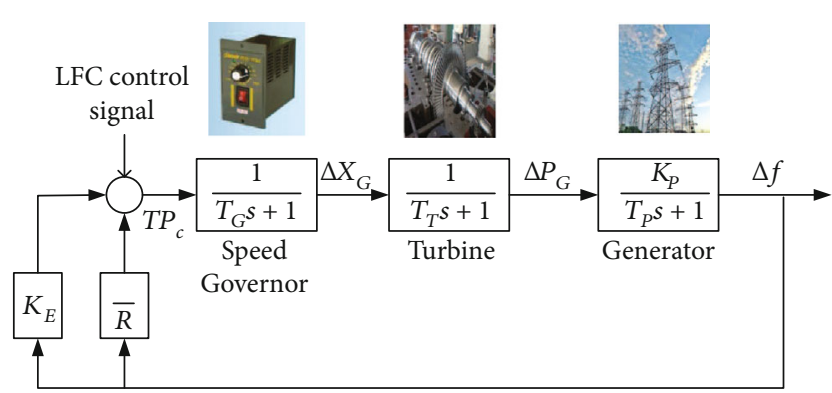

FIGURE 5: Block diagram of an LFC system for power grid.

where

$$
\begin{gathered}
\bar{B}_{J}=\left[\begin{array}{cc}
B_{J} & 0 \\
0 & 0
\end{array}\right], \bar{B}=\left[\begin{array}{ll}
B & 0 \\
0 & 0
\end{array}\right], \\
E_{i}=\left[\begin{array}{cc}
V_{i} & W_{i 2} \\
0 & 0
\end{array}\right], F_{i}=\left[\begin{array}{c}
W_{i 1} \\
1
\end{array}\right] .
\end{gathered}
$$

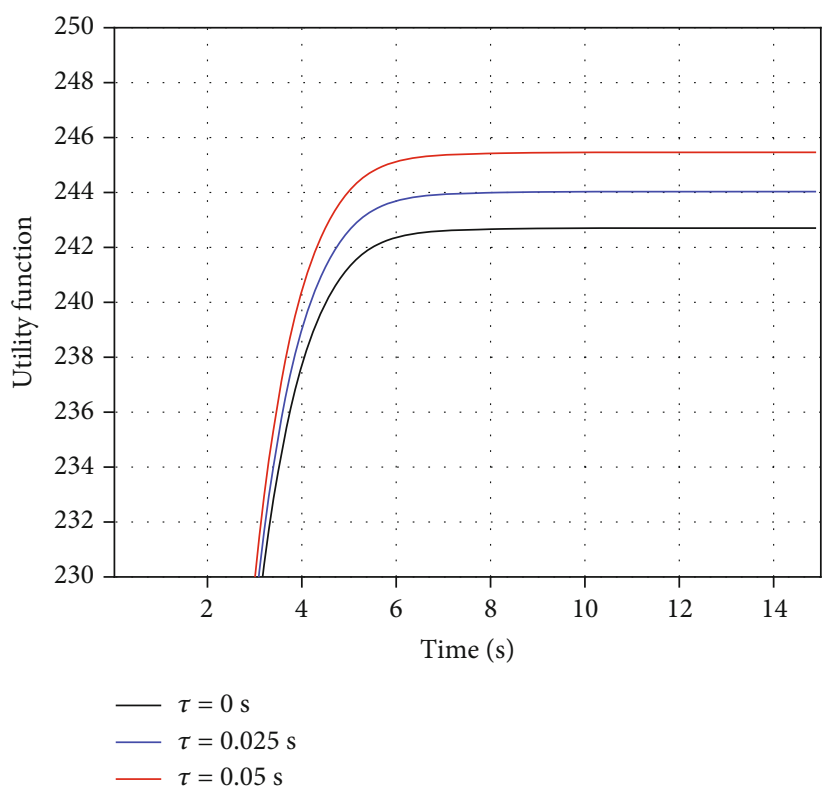

Figure 6: Performance comparison of different time delays with the sampling period $T=0.1 \mathrm{~s}$. 


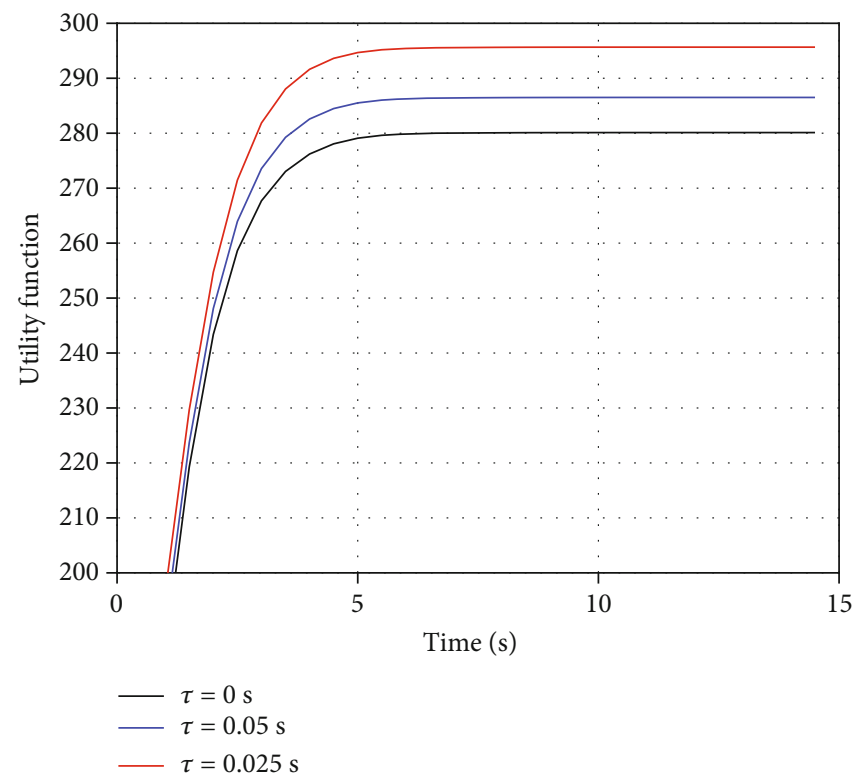

FIgURE 7: Performance comparison of different time delays with the sampling period $T=0.5 \mathrm{~s}$.

problem (23) is given by

$$
u_{\widehat{k}, i}^{*}=-H_{\widehat{k}, i} f_{\widehat{k}, i}, i=1,2, \cdots, J-1 \text {, }
$$

where

$$
\begin{gathered}
H_{\widehat{k}, i}=\left[F_{i}^{T} Q_{i+1} F_{i}+C\right]^{-1} F_{i}^{T} Q_{i+1} E_{i}, \\
Q_{i}=E_{i}^{T} Q_{i+1} E_{i}+\bar{B}-H_{\widehat{k}, i}^{T} F_{i}^{T} Q_{i+1} E_{i}, \\
Q_{J}=\bar{B}_{J} .
\end{gathered}
$$

The proof can be achieved similar to the derivation process of optimal control strategy in [37].

3.4. Joint Optimal Path Determination. Once the set of candidate transmission paths and the corresponding optimal control design are determined, the utility function can be expressed as

$$
P_{J}^{\widehat{k}}\left(u_{\widehat{k}, i}^{*}\right)=f_{\widehat{k}, J}^{T} \bar{B}_{J} f_{\widehat{k}, J}+\sum_{i=0}^{J-1}\left\{f_{\widehat{k}, i}^{T} \bar{B} f_{\widehat{k}, i}+\left(u_{k \wedge, i}^{*}\right)^{T} C u_{\widehat{k}, i}^{*}\right\} .
$$

Then, the best transmission path is determined by

$$
k^{*}=\arg \min _{\{\widehat{k}\}}\left\{P_{J}^{\widehat{k}}\left(u_{\widehat{k}, i}^{*}\right)\right\}
$$

and the corresponding optimal control design is given by (24) that

$$
u_{k^{*}, i}^{*}=-H_{k^{*}, i} f_{k^{*}, i}
$$

Therefore, in order to meet the requirements of real-time control and efficient power consumption, the three-step joint optimization algorithm can be summarized as in Algorithm 2. First, the set of candidate transmission paths is obtained by using the minimum hop routing algorithm, and then, the optimal control design for each candidate transmission path can be derived in a backward recursion manner. Finally, the best transmission path and the corresponding control design are determined by the minimum utility function.

\section{Simulation Results}

In this section, a case study of the load frequency control (LFC) system in power grid [37] is used to verify the performance of the provided joint optimal design for WSANs. In the simulation, 15 sensor nodes are considered in the shared wireless network, and the controller is placed in a determined location. The distance between sensor nodes is uniform in $[1 \mathrm{~m}, 5 \mathrm{~m}]$.

The typical LFC system consists of generator, turbine, speed governor, and LFC controllers as shown in Figure 5. The objective is to design the control signal, namely, the speed, to maintain the frequency deviation $\Delta f$ within the specified range. The plant state is $s(t)=\left[\begin{array}{llll}\Delta P_{c} & \Delta f & \Delta P_{G} & \Delta X_{G}\end{array}\right]^{T}$, and $\Delta X_{G}, \Delta P_{G}$, and $\Delta P_{c}$ represent the valve position, the derivation of generator mechanical output, and the generator output, respectively [37]. The system parameters are given by

$$
\begin{gathered}
W=\left[\begin{array}{cccc}
0 & 1 & 0 & 0
\end{array}\right], \\
V=\left[\begin{array}{cccc}
0 & -1 & 0 & 1 \\
0 & 0 & 0 & 0 \\
0 & 0 & 0 & -1 \\
0 & 0 & 0.95 & -1.2
\end{array}\right] .
\end{gathered}
$$




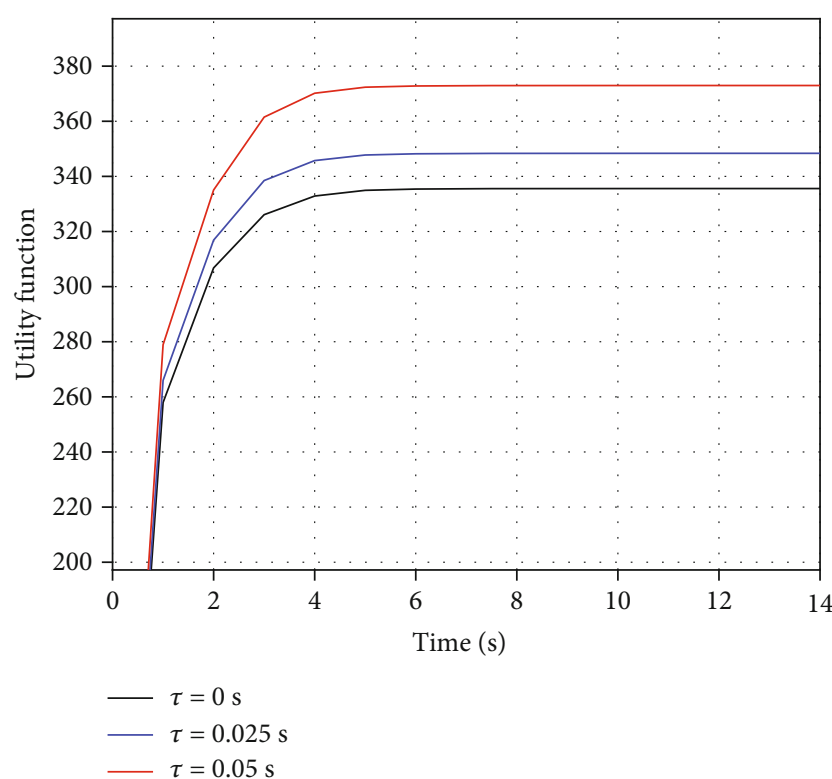

FIgURe 8: Performance comparison of different time delays with the sampling period $T=1.0 \mathrm{~s}$.

In the simulation, we set the weight coefficient $\beta=1$, and

$$
B_{J}=B_{0}=\left[\begin{array}{llll}
1 & 0 & 0 & 0 \\
0 & 1 & 0 & 0 \\
0 & 0 & 1 & 0 \\
0 & 0 & 0 & 1
\end{array}\right], C_{0}=1
$$

First, the utility functions of the proposed joint optimization algorithm in the presence of different time delays are shown in Figures 6-8. The sampling periods are set as $T=$ $0.1 \mathrm{~s}, 0.5 \mathrm{~s}, 1.0 \mathrm{~s}$ with different time delays $\tau=0,0.25 T, 0.5 \mathrm{~T}$ in different scenarios. It can be seen from figures that the utility function can gradually converge to a constant value, which indicates that the proposed joint optimization algorithm is efficient and stable under various time delays. Furthermore, the comparison results show that the time delay will influence the utility function. That is, as the time delay increases, the utility function becomes larger, which means that more serious the system performance degradation is caused.

In order to further verify the effectiveness of the proposed joint optimization algorithm, the convergence utility function is shown in Figure 9 when the ratio of the time delay to the sampling interval is set from 0 to 0.9 . It can be seen that the system stability can always be guaranteed. In addition, the lower utility function can be obtained either the sampling interval or the time delay becomes larger. This stems from the fact that increasing the sampling interval and time delay will delay the execution of the control signal and also slow down the acquisition frequency of the plant state, which makes it much more difficult for the plant to converge.

Finally, in Figures 10-12, we show performance comparison in three different cases: individual optimal transmission path routing, individual optimal control strategy design, and

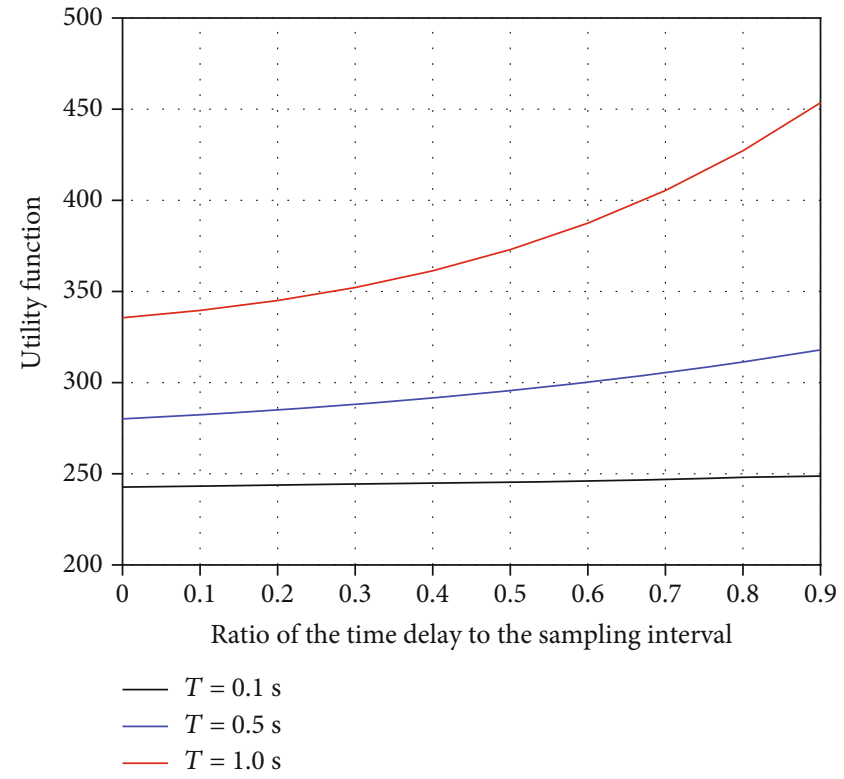

FIgURe 9: Performances of the proposed algorithm with different time delays.

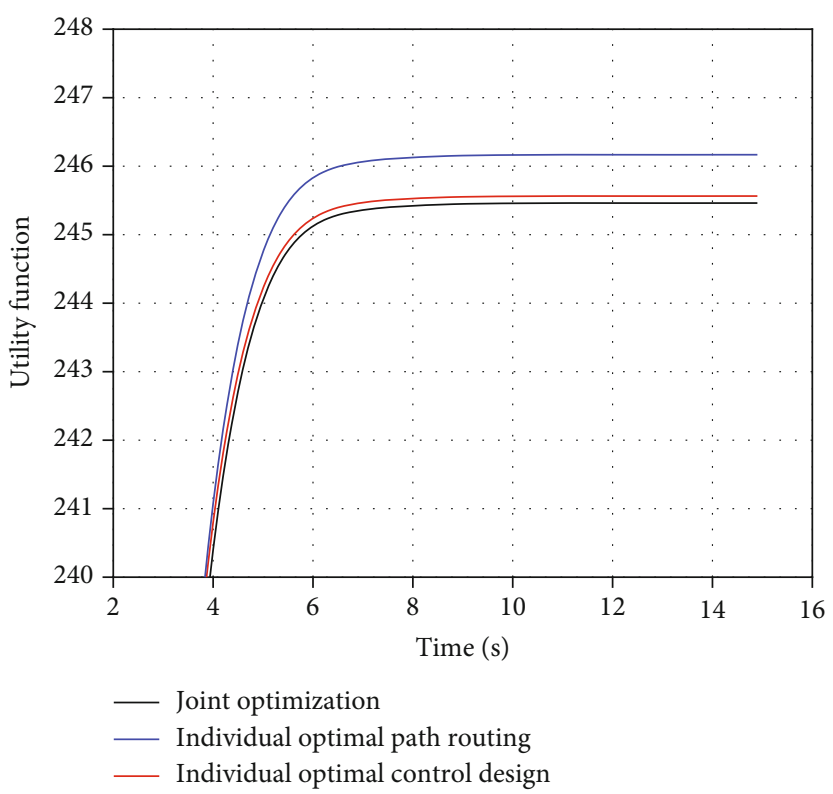

FIGURE 10: Utility function comparison of three algorithms with the sampling period $T=0.1 \mathrm{~s}$.

the proposed joint optimization. The sampling periods are set as $T=0.1 \mathrm{~s}, 0.5 \mathrm{~s}, 1.0 \mathrm{~s}$ and the time delay is $\tau=0.5 T$. The results indicate that the proposed joint optimization design is superior to the independent design. The joint optimization scheme improves the stability of the control system and the power consumption efficiency. We can also observe that, when the delay is relatively small, the performance of the individual transmission path routing is very close to the performance of the joint optimization algorithm. While its utility function will significantly increase, even fail to converge, when the time delay becomes larger. 


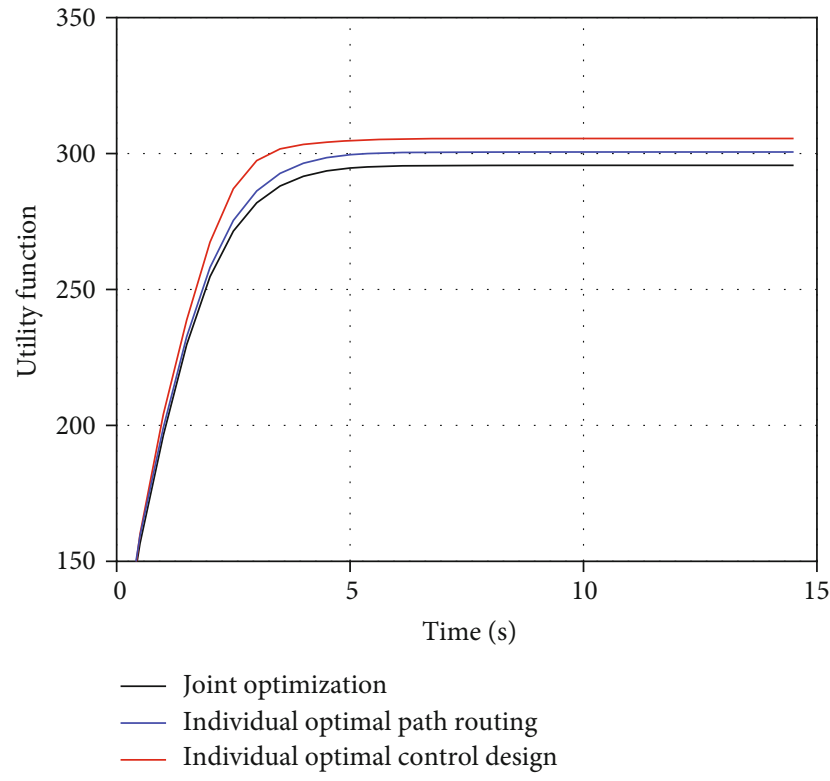

FIGURE 11: Utility function comparison of three algorithms with the sampling period $T=0.5 \mathrm{~s}$.

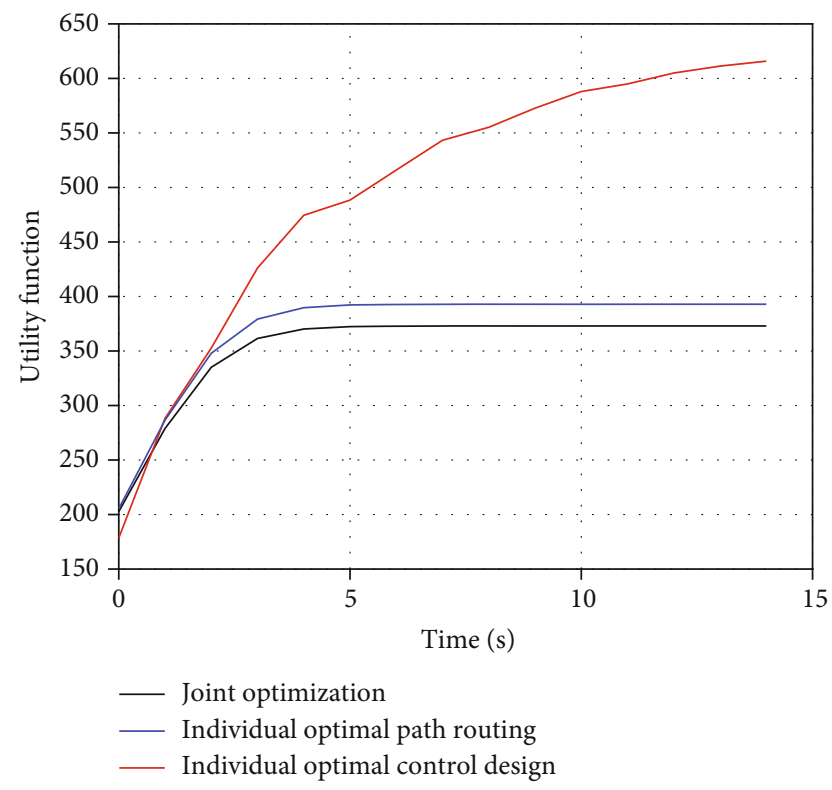

FIGURE 12: Utility function comparison of three algorithms with the sampling period $T=1.0 \mathrm{~s}$.

\section{Conclusions}

In this paper, an improved joint optimization scheme of the WSAN system is proposed taking into account the networkinduced time delays caused by wireless communication. The WSAN is modeled as a linear system in discrete-time domain and the joint optimization problem is formulated as a quadratic utility function, which can be decomposed into two subproblems, and then, a three-step algorithm is designed in the closed-loop feedback control. Finally, a case study of the LFC in power grid system is investigated to demonstrate the effectiveness of the proposed joint optimization algorithm that better control stability and power consumption efficiency are achieved.

\section{Data Availability}

The data used to support the findings of this study are available from the corresponding author upon request.

\section{Conflicts of Interest}

The authors declare that there is no conflict of interest regarding the publication of this paper.

\section{Acknowledgments}

This work was supported by the National Natural Science Foundation of China (62001011), the Beijing Wuzi Youth Foundation (2020XJQN08), the Beijing Natural Science Foundation (L202016), the Scientific Research Plan of Beijing Municipal Commission of Education (KM201910005026), and the Beijing Nova Program of Science and Technology (Z191100001119094).

\section{References}

[1] Y. Mao, C. You, J. Zhang, K. Huang, and K. B. Letaief, “A survey on mobile edge computing: the communication perspective," IEEE Communications Surveys \& Tutorials, vol. 19, no. 4, pp. 2322-2358, 2017.

[2] J. Xiong, J. Ren, L. Chen et al., "Enhancing privacy and availability for data clustering in intelligent electrical service of IoT," IEEE Internet of Things Journal, vol. 6, no. 2, pp. 15301540, 2019.

[3] D. Wu, R. Bao, Z. Li, H. Wang, H. Zhang, and R. Wang, "Edgecloud collaboration enabled video service enhancement: a hybrid human-artificial intelligence scheme," IEEE Transactions on Multimedia, vol. 23, pp. 2208-2221, 2021.

[4] N. Primeau, R. Falcon, R. Abielmona, and E. M. Petriu, "A review of computational intelligence techniques in wireless sensor and actuator networks," IEEE Communications Surveys \& Tutorials, vol. 20, no. 4, pp. 2822-2854, 2018.

[5] L. Da Xu, W. He, and S. Li, "Internet of things in industries: a survey," IEEE Transactions on Industrial Informatics, vol. 10, no. 4, pp. 2233-2243, 2014.

[6] J. Xiong, M. Zhao, M. Z. A. Bhuiyan, L. Chen, and Y. Tian, “An AI-enabled three-party game framework for guaranteed data privacy in mobile edge crowdsensing of IOT," IEEE Transactions on Industrial Informatics, vol. 17, no. 2, pp. 922-933, 2021.

[7] X. Xue and J. Zhang, "Matching large-scale biomedical ontologies with central concept based partitioning algorithm and adaptive compact evolutionary algorithm," Applied Soft Computing, vol. 106, article 107343, 2021.

[8] X. Xue, C. Yang, C. Jiang, P.-W. Tsai, G. Mao, and H. Zhu, "Optimizing ontology alignment through linkage learning on entity correspondences," Complexity, vol. 2021, Article ID 5574732, 12 pages, 2021.

[9] Z. Wang, Y. Gao, C. Fang, L. Liu, H. Zhou, and H. Zhang, "Optimal control design for connected cruise control with 
stochastic communication delays," IEEE Transactions on Vehicular Technology, vol. 69, no. 12, pp. 357-369, 2020.

[10] D. Wu, X. Han, Z. Yang, and R. Wang, "Exploiting transfer learning for emotion recognition under cloud-edge-client collaborations," IEEE Journal on Selected Areas in Communications, vol. 39, no. 2, pp. 479-490, 2020.

[11] F. Xia, "QoS challenges and opportunities in wireless sensor/ actuator networks," Sensors, vol. 8, no. 2, pp. 1099-1110, 2008.

[12] J. Xiong, R. Ma, L. Chen et al., "A personalized privacy protection framework for mobile crowdsensing in IIoT," IEEE Transactions on Industrial Informatics, vol. 16, no. 6, pp. 4231-4241, 2020.

[13] I. F. Akyildiz and I. H. Kasimoglu, "Wireless sensor and actor ${ }^{\text {is }}$ networks: research challenges," Ad Hoc Networks, vol. 2, no. 4, pp. 351-367, 2004.

[14] X. Xue, X. Wu, C. Jiang, G. Mao, and H. Zhu, "Integrating sensor ontologies with global and local alignment extractions," Wireless Communications and Mobile Computing, vol. 2021, Article ID 6625184, 10 pages, 2021.

[15] L. Zhang, H. Gao, and O. Kaynak, "Network-induced constraints in networked control systems-a survey," IEEE Transactions on Industrial Informatics, vol. 9, no. 1, pp. 403-416, 2013.

[16] J. Nilsson, B. Bernhardsson, and B. Wittenmark, "Stochastic analysis and control of real-time systems with random time delays," Automatica, vol. 34, no. 1, pp. 57-64, 1998.

[17] H. Shousong and Z. Qixin, "Stochastic optimal control and analysis of stability of networked control systems with long delay," Automatica, vol. 39, no. 11, pp. 1877-1884, 2003.

[18] X.-M. Zhang, Q.-L. Han, A. Seuret, F. Gouaisbaut, and Y. He, "Overview of recent advances in stability of linear systems with timevarying delays," IET Control Theory \& Applications, vol. 13, no. 1, pp. 1-16, 2019.

[19] R. Deng, R. Lu, C. Lai, T. H. Luan, and H. Liang, “Optimal workload allocation in fog-cloud computing toward balanced delay and power consumption," IEEE Internet of Things Journal, vol. 3, no. 6, pp. 1171-1181, 2016.

[20] A. Yousefpour, G. Ishigaki, and J. P. Jue, "Fog computing: Towards minimizing delay in the internet of things," in 2017 IEEE international conference on EDGE computing (EDGE), pp. 17-24, IEEE, Honolulu, HI, USA, 2017.

[21] Y.-B. Zhao, G.-P. Liu, Y. Kang, and L. Yu, "Stochastic stabilization of packet-based networked control systems," in PacketBased Control for Networked Control Systems, pp. 77-85, Springer, 2018.

[22] Z. Wang, Y. Guo, Y. Gao, C. Fang, M. Li, and E. Sun, "Joint optimization of control law and power consumption for wireless sensor and actuator networks," in 2019 IEEE Global Communications Conference (GLOBECOM), pp. 1-6, IEEE, Waikoloa, HI, USA, 2019.

[23] J. Aponte-Luis, J. Gómez-Galán, F. Gómez-Bravo, M. SánchezRaya, J. Alcina-Espigado, and P. Teixido-Rovira, "An efficient wireless sensor network for industrial monitoring and control," Sensors, vol. 18, no. 2, p. 182, 2018.

[24] C. S. Abella, S. Bonina, A. Cucuccio et al., "Autonomous energy-efficient wireless sensor network platform for home/ office automation," IEEE Sensors Journal, vol. 19, no. 9, pp. 3501-3512, 2019.

[25] Z. Li, Y. Jiang, Y. Gao, L. Sang, and D. Yang, "On bufferconstrained throughput of a wireless-powered communication system," IEEE Journal on Selected Areas in Communications, vol. 37, no. 2, pp. 283-297, 2019.
[26] M. Kubisch, H. Karl, A. Wolisz, L. C. Zhong, and J. Rabaey, "Distributed algorithms for transmission power control in wireless sensor networks," in 2003 IEEE Wireless Communications and Networking, 2003. WCNC 2003, pp. 558-563, New Orleans, LA, USA, 2003.

[27] M. J. Miller and N. H. Vaidya, "A mac protocol to reduce sensor network energy consumption using a wakeup radio," IEEE Transactions on Mobile Computing, vol. 4, no. 3, pp. 228-242, 2005.

[28] Y. Sadi, S. C. Ergen, and P. Park, "Minimum energy data transmission for wireless networked control systems," IEEE Transactions on Wireless Communications, vol. 13, no. 4, pp. 2163 2175, 2014.

[29] Y. Jiang, Q. Liu, F. Zheng, X. Gao, and X. You, "Energy-efficient joint resource allocation and power control for D2D communications," IEEE Transactions on Vehicular Technology, vol. 65, no. 8, pp. 6119-6127, 2016.

[30] Q. Jin, G. Wu, K. Boriboonsomsin, and M. J. Barth, "Powerbased optimal longitudinal control for a connected ecodriving system," IEEE Transactions on Intelligent Transportation Systems, vol. 17, no. 10, pp. 2900-2910, 2016.

[31] J. Ren, G. Yu, Y. Cai, and Y. He, "Latency optimization for resource allocation in mobile-edge computation offloading," IEEE Transactions on Wireless Communications, vol. 17, no. 8, pp. 5506-5519, 2018.

[32] H. Al-Tous and I. Barhumi, "Differential game for resource allocation in energy harvesting wireless sensor networks," IEEE Transactions on Green Communications and Networking, vol. 4, no. 4, pp. 1165-1173, 2020.

[33] R. A. Gupta and M.-Y. Chow, "Networked control system: overview and research trends," IEEE Transactions on Industrial Electronics, vol. 57, no. 7, pp. 2527-2535, 2009.

[34] X. Ge, F. Yang, and Q.-L. Han, "Distributed networked control systems: a brief overview," Information Sciences, vol. 380, pp. 117-131, 2017.

[35] L. Mo, X. Cao, Y. Song, and A. Kritikakou, "Distributed node coordination for real-time energy-constrained control in wireless sensor and actuator networks," IEEE Internet of Things Journal, vol. 5, no. 5, pp. 4151-4163, 2018.

[36] W. B. Heinzelman, A. P. Chandrakasan, and H. Balakrishnan, "An application-specific protocol architecture for wireless microsensor networks," IEEE Transactions on Wireless Communications, vol. 1, no. 4, pp. 660-670, 2002.

[37] Z. Wang, X. Wang, L. Liu, and M. Huang, "Optimal state feedback control for wireless networked control systems with decentralised controllers," IET Control Theory \& Applications, vol. 9, no. 6, pp. 852-862, 2015.

[38] R. Sriram, G. Manimaran, and C. S. R. Murthy, "Preferred link based delay-constrained least-cost routing in wide area networks," Computer Communications, vol. 21, no. 18, pp. 1655-1669, 1998.

[39] S. Ping, "Delay measurement time synchronization for wireless sensor networks," Intel Research Berkeley Lab, vol. 6, pp. 1-10, 2003.

[40] S. Lindsey, C. Raghavendra, and K. M. Sivalingam, "Data gathering algorithms in sensor networks using energy metrics," IEEE Transactions on Parallel \& Distributed Systems, vol. 9, pp. 924-935, 2002.

[41] M. Haenggi and D. Puccinelli, "Routing in ad hoc networks: a case for long hops," IEEE Communications Magazine, vol. 43, no. 10, pp. 93-101, 2005. 